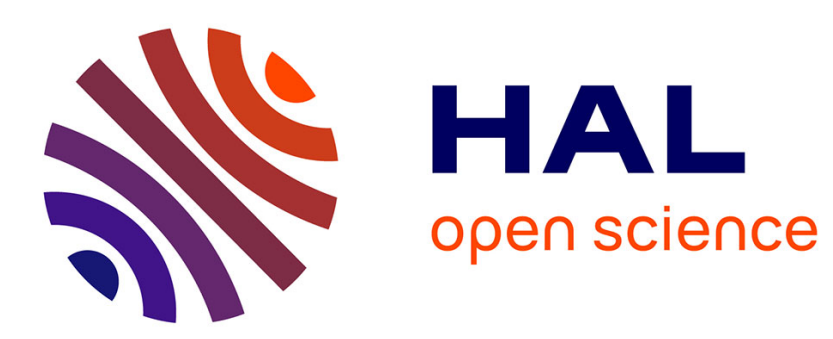

\title{
Generalized boundary condition for multiple scatter in acoustic reflection
}

Maurice A. Biot

\section{To cite this version:}

Maurice A. Biot. Generalized boundary condition for multiple scatter in acoustic reflection. Journal of the Acoustical Society of America, 1968, 44 (6), pp.1616-1622. 10.1121/1.1911304 . hal-01368601

\section{HAL Id: hal-01368601 \\ https://hal.science/hal-01368601}

Submitted on 21 Sep 2016

HAL is a multi-disciplinary open access archive for the deposit and dissemination of scientific research documents, whether they are published or not. The documents may come from teaching and research institutions in France or abroad, or from public or private research centers.
L'archive ouverte pluridisciplinaire HAL, est destinée au dépôt et à la diffusion de documents scientifiques de niveau recherche, publiés ou non, émanant des établissements d'enseignement et de recherche français ou étrangers, des laboratoires publics ou privés. 


\title{
Generalized Boundary Condition for Multiple Scatter in Acoustic Reflection
}

\author{
M. A. BIoT* \\ Cornell Aeronautical Laboratory, Inc., \\ PO Box 235, Buffalo, New York 14221
}

\begin{abstract}
A boundary condition is developed that generalizes the author's earlier results and embodies the effect of multiple scatter for acoustic reflection on a rough surface. The size of the roughness is assumed to be sufficiently small in comparison with the wavelength. Both induced radiating dipoles and radiating sources are taken into account. The results are further generalized to take into account roughness of various shapes, nonuniform distribution, and anisotropic properties. The anisotropy is represented by a two-dimensional symmetric tensor of Rank 2. Results open the way to the analysis of combined coherent and incoherent reflection including the cooperative resonance due to multiple scatter.
\end{abstract}

\section{INTRODUCTION}

$I^{\mathrm{N}}$ $\mathrm{N}$ some earlier work, the author has shown that the effect of roughness on the reflection of acoustic and electromagnetic waves including the influence of multiple scatter could be obtained under certain conditions by satisfying a suitable boundary condition on a smooth surface. The analysis for the acoustic problem was developed in two papers, ${ }^{1,2}$ where the roughness is represented by a uniform distribution of hemispherical bosses all of the same size, and the equivalent boundary condition is obtained by introducing a suitable distribution of radiating dipoles.

The purpose of the present paper is first to complete the theory by taking into account not only the induced dipoles, but also the induced sources. In addition, the theory is further generalized to include roughness elements of various closeness and shapes, anisotropic properties and nonuniform distribution.

Results of the theory for the case of nonuniform distribution of the roughness opens the way to the analysis of reflected waves containing both coherent and noncoherent components including the cooperative resonance characteristic of multiple scatter. This is of particular interest in applications to the case where the

\footnotetext{
* Consultant, Cornell Aeron. Lab., Inc.

1 M. A. Biot, "Reflection on a Rough Surface from an Acoustic Point Source," J. Acoust. Soc. Amer. 29, 1193-1200 (1957).

2 M. A. Biot, "On the Reflection of Acoustic Waves on a Rough Surface," J. Acoust. Soc. Amer. 30, 479-480 (1958).
}

roughness distribution possesses a correlation distance of the order of the wavelength.

The theory leads to a very general boundary condition that represents the combined effect of nonuniformity and anisotropic properties of the roughness. A feature essentially due to multiple scatter and brought out by the introduction of a close interaction factor is the anisotropic reflection that results from anisotropic spacing of hemispherical bosses.

The acoustic theory is an outgrowth of similar boundary conditions developed earlier for multiple scatter of electromagnetic waves. ${ }^{3,4}$

Note that the boundary condition provides essentially the magnitudes of scattering sources and dipoles as if they were continuously distributed. The actual scatter both coherent and incoherent is then readily evaluated by a standard summation process applied to the discrete scatterers. An approximation to the coherent reflection may also be obtained by retaining a continuous distribution of scatterers. In this case, the reflection occurs only with a change of phase as shown in Sec. 4.

The boundary condition derived here is applicable to nonuniform roughness whose distribution is represented by Fourier components of wavelength significantly larger than the scatterers, but may be comparable to

\footnotetext{
3. A. Biot, "Some New Aspects of the Reflection of Electromagnetic Waves on a Rough Surface," J. Appl. Phys. 28, 14551463 (1957).

"M. A. Biot, "On the Reflection of Electromagnetic Waves on a Rough Surface," J. Appl. Phys. 29, 998 (1958).
} 
the wavelength of the incident wave. This yields a nonspecular reflection and scatter superposed on the smaller scale scatter due to the roughness. A problem that bears some resemblance to this was considered by Semyonov ${ }^{5}$ for the scatter of electromagnetic waves due to small roughness on an uneven surface, where the characteristic dimension of the latter is large compared to the wavelength.

Nonspherical scatterers of a very general nature have also been considered by Twersky ${ }^{6}$ under the assumption of farfield interaction of the scatterers. The twodimensional problem of scatter by parallel elliptic cylinders on a plane surface was also treated by Burke and Twersky ${ }^{7}$ for wavelengths both large and small relative to the size of the scatterers.

\section{ISOLATED SCATTERING CENTER}

Consider a solid sphere of radius $a$ subject to an incident acoustic wave. The center of the sphere is located at the origin. We denote by $U$ the velocity field of the incident wave in the absence of the solid sphere.

When the wavelength is large relative to the radius of the sphere it is well known that part of the scattered wave is represented by a radiating dipole of velocity potential:

$$
\varphi_{d}=-\frac{1}{2} a^{3} \mathbf{U}_{0} \cdot \nabla F
$$

where $\mathrm{U}_{0}$ is the velocity of the incident wave at the origin, and

$$
\begin{aligned}
& \nabla=\mathrm{i} \partial / \partial x+\mathrm{j} \partial / \partial y+\mathrm{k} \partial / \partial z, \\
& F=e / R^{-i k R} \\
& R=\left(x^{2}+y^{2}+z^{2}\right)^{1} .
\end{aligned}
$$

The wavenumber is

$$
k=\omega / c
$$

with $\omega$ the angular frequency and $c$ the velocity of sound. The time factor $\exp (i \omega t)$ is omitted.

The velocity field of the scattered wave due to the dipole is then given by $\nabla \varphi_{d}$.

As is shown below, it is necessary to take into account another scattered wave corresponding to a radiating source in contrast to a dipole.

The radiating source is derived by considering the volume change of the incident wave. The relative rate of change of volume of the incident wave at the origin is

$$
D_{0}=\boldsymbol{\nabla} \cdot \mathbf{U} \text {. }
$$

This represents the value at the origin of the divergence of the field $\mathbf{U}$. Hence the rate of volume outflow of the

\footnotetext{
5 B. I. Semyonov, "Approximate Computation of Scattering of Electromagnetic Waves by Rough Surface Contours," Radio Eng. Electron. Phys. 11, 1179-1187 (1966) (transl.).

'V. Twersky, "Acoustic Bulk Parameters of Random Volume Distributions of Small Scatterers," J. Acoust. Soc. Amer. 36, 13141329 (1964).

7 J. E. Burke and V. Twersky, "Scattering and Reflection by Elliptically Striated Surfaces," J. Acoust. Soc. Amer. 40, 883-895 (1966).
}

field $\mathbf{U}$ across the surface of the sphere of radius $a$ is

$$
Q=(4 / 3) \pi a^{3} D_{0} \text {. }
$$

Hence, the presence of a solid sphere of this radius requires the cancellation of this outflow by placing at the center a radiating source of velocity potential:

$$
\varphi_{s}=\frac{1}{3} a^{3} D_{0} F \text {. }
$$

The outflow of this source is equal to $-Q$. The total velocity potential $\varphi$ due to the source and dipole is derived by adding the values obtained from Eqs. 1a and 2e. Hence,

$$
\varphi=\varphi_{d}+\varphi_{s}=-\frac{1}{2} a^{3} \mathrm{U}_{0} \cdot \nabla F+\frac{1}{3} a^{3} D_{0} F .
$$

Consider the case of a plane incident wave propagating in the $x$ direction. The $x$ component of the velocity field of the incident wave may be written

$$
U_{x}=U_{0} \exp (i k x) .
$$

The divergence of this field is

$$
d U_{x} / d x=i k U_{0} \exp (i k x),
$$

and its value at the origin is

$$
D_{0}=i k U_{0} .
$$

Hence, the velocity potential (Eq. 2f) of the scattered wave is

$$
\varphi=-\frac{1}{2} a^{3} U_{0} \partial F / \partial x+\frac{1}{3} i k a^{3} U_{0} F .
$$

At large distance, this potential becomes

$$
\varphi=\frac{1}{3} i k a^{3} U_{0} F[1+(3 / 2)(x / R)] .
$$

Hence, the energy radiated by the dipole and the source are of the same order, and both must be taken into account for a correct evaluation of the scattered field.

Expression 1k coincides with the result obtained by Lord Rayleigh. ${ }^{8}$

\section{CONTINUOUS DISTRIBUTION OF RADIATING DIPOLES AND SOURCES}

If, in Expression 1f, we put

$$
\begin{aligned}
& \mathbf{M}=-\frac{1}{2} a^{3} \mathrm{U}_{0}, \\
& M=\frac{1}{3} a^{3} D_{0},
\end{aligned}
$$

the potential of the scattered field becomes

$$
\varphi=\mathbf{M} \cdot \boldsymbol{\nabla} F+M F .
$$

This represents the field radiated by a dipole and a source without any reference to the manner by which they are generated. The magnitude of the dipole is measured by the vector $\mathbf{M}$ and $M$ measures the magnitude of the source. They are both located at the origin.

In order to analyze the reflective properties of a rough surface, we first consider that the radiating dipoles

${ }^{8} \mathrm{~J}$. W. Strutt, Lord Rayleigh, The Theory of Sound (Dover Publications, Inc., New York, 1945), Vol. II, p. 276. 
and sources are continuously distributed over the $x-y$ plane. The dipoles are also assumed to be oriented in the same plane. Consider a dipole located at the point $x=\xi, y=\eta$. The magnitude of this dipole is determined by the vector

$$
\mathrm{M}=M_{x} \mathbf{i}+M_{y} \mathbf{j},
$$

and the magnitude of the source is $M$. According to Eq. 3b, the velocity potential of the field radiated by this dipole and this source is

$$
\varphi=M_{x} \partial F / \partial x+M_{y} \partial F / \partial y+M F .
$$

In the value of $F$ we must now replace $R$ by

$$
R=\left[(x-\xi)^{2}+(y-\eta)^{2}+z^{2}\right]^{t} .
$$

Hence, $\varphi$ may also be written

$$
\varphi=-M_{x} \partial F / \partial \xi-M_{y} \partial F / \partial \eta+M F .
$$

We now assume that the dipoles and sources are continuously distributed over the $x-y$ plane with magnitudes and orientation dependent on the location. The quantities $M_{x}(\xi, \eta), M_{y}(\xi, \eta)$, and $M(\xi, \eta)$ are now functions of $\xi$ and $\eta$. If the distance between these radiating singularities is sufficiently small the total radiated field may be obtained by assuming a continuous distribution. If there are $N$ dipoles per unit area, the vector density

$$
\begin{aligned}
& \mu_{x}=N M_{x}(\xi, \eta), \\
& \mu_{y}=N M_{y}(\xi, \eta),
\end{aligned}
$$

measures the dipole magnitude per unit area. For the same number $N$ of sources per unit area, their surface density is

$$
\mu=N M(\xi, \eta) \text {. }
$$

The total field radiated by such a continuous distribution of dipoles and sources over the area $S$ is obtained by surface integration of the potential (Eq. 3f) where densities are substituted in place of $M_{x}, M_{y}$, and $M$. The velocity potential of this total field is

$$
\phi_{s}=\iint_{S}\left(-\mu_{x} \frac{\partial F}{\partial \xi}-\mu_{y} \frac{\partial F}{\partial \eta}+\mu F\right) d \xi d \eta .
$$

Integration by parts yields

$$
\phi_{s}=\iint_{S} \zeta F d \xi d \eta+\int_{C} F\left(-\mu_{x} d \eta+\mu_{y} d \xi\right)
$$

with

$$
\zeta=\partial \mu_{x} / \partial \xi+\partial \mu_{y} / \partial \eta+\mu .
$$

The line integral is evaluated clockwise on the closed contour $C$, which contains the area $S$.

As can be seen, the scattered field is the same as if sources were distributed continuously over the area $S$ and on its boundary $C$. For such a surface distribution of sources, there is a well-known relation, derived from Gauss's theorem, which relates the surface density $\zeta$ of the sources to the normal derivative of the potential. In the present case, this relation is

$$
\partial \phi_{s} / \partial z=-2 \pi \xi
$$

The normal derivative $\partial \phi_{s} / \partial z$ must be evaluated on the side $z>0$ of the surface. Using the value obtained from Eq. $3 \mathrm{k}$ for $\zeta$, we substitute the variables $x, y$, instead of $\xi, \eta$, and write Relation 31 as

$$
\partial \phi_{s} / \partial z=-2 \pi\left(\partial \mu_{x} / \partial x+\partial \mu_{y} / \partial y\right)-2 \pi \mu .
$$

This fundamental relation, at any point $x, y$ of the surface, between the normal derivative of the field and the local density of the dipoles and sources is now used in deriving the acoustic properties of a rough surface. It is shown that under certain conditions the effect of the roughness on acoustic reflection may be obtained by applying a modified boundary condition to a smooth surface.

\section{EFFECT OF ROUGHNESS REPLACED BY A BOUNDARY CONDITION}

Consider a rigid reflecting surface coinciding with the $x-y$ plane and an acoustic medium located in the half space $z>0$. An incident and reflected acoustic wave, whether plane or emanating from any set of sources in the acoustic medium, is represented by a velocity potential $\phi_{1}$. The boundary condition satisfied by this potential at the plane boundary $(z=0)$ is

$$
\partial \phi_{1} / \partial z=0 .
$$

A simple model for a rough surface may be obtained by a distribution of rigid hemispherical bosses over the reflecting plane. The centers of these bosses are located in the $x-y$ plane, and the average number per unit area is assumed to be a constant value $N$. It is further assumed that the radius as well as the mutual distances of these bosses is small compared to the wavelength. In this case, the effect of the roughness is to add to the undisturbed field $\phi_{1}$ a field $\phi_{s}$ radiated by a distribution of dipoles and sources. The field $\phi_{s}$ is given by Expression $3 \mathrm{i}$. The density distribution $\mu_{x}, \mu_{y}$, and $\mu$ of the dipoles and sources is yet unknown. The total acoustic field including the effect of the roughness is

$$
\phi=\phi_{1}+\phi_{s} \text {. }
$$

If we take into account the condition of Eq. $4 \mathrm{a}$, we have

$$
\partial \phi / \partial z=\partial \phi_{s} / \partial z
$$

Hence, Relation $3 \mathrm{~m}$ becomes

$$
\partial \phi / \partial z=-2 \pi\left(\partial \mu_{x} / \partial x+\partial \mu_{y} / \partial y\right)-2 \pi \mu .
$$

It remains now to relate the unknown density distributions $\mu_{x}, \mu_{y}$, and $\mu$ to the unknown field $\phi$. This can be done as follows.

Consider the hemispherical boss of radius $a$ centered at the point $x, y$ on the reflecting plane. We shall assume that in the absence of the boss the velocity field 


\section{BOUNDARY CONDITION}

in a region of size $2 a$ in the vicinity of the point $x, y$ is approximately uniform. This uniform velocity field is parallel to the plane of reflection and may be represented by the two components $U_{x}$ and $U_{y}$ along the $x$ and $y$ directions. This velocity plays the rôle of $\mathbf{U}_{0}$ in Eq. 3a. Hence, the induced dipole due to the hemispherical boss located at the point $x, y$ is represented by a vector $\mathbf{M}$ lying in the $x-y$ plane of components :

$$
\begin{aligned}
& M_{x}=-\frac{1}{2} a^{3} U_{x}, \\
& M_{y}=-\frac{1}{2} a^{3} U_{y} .
\end{aligned}
$$

Similarly, the source corresponding to the hemispherical boss is given by

$$
M=\frac{1}{3} a^{3} D,
$$

where $D$ is the divergence of the velocity field at the point $x, y$ in the absence of the boss.

Combining Eqs. $3 \mathrm{~g}$ and $3 \mathrm{~h}$ with Eqs. $4 \mathrm{e}$ and 4 f, we write for the density distributions

$$
\begin{aligned}
\mu_{x} & =-\frac{1}{2} a^{3} N U_{x}, \\
\mu_{y} & =-\frac{1}{2} a^{3} N U_{y}, \\
\mu & =\frac{1}{3} a^{3} N D .
\end{aligned}
$$

The last step is to relate $U_{x}, U_{y}$, and $D$ to the unknown field.

If the bosses and their mutual distances are sufficiently small relative to the wavelength and if at the same time the bosses are not too close together, we may assume that $U_{x}$ and $U_{y}$ are velocities of the total field $\phi$ at $z=0$. Hence,

$$
\begin{aligned}
& U_{x}=\partial \phi / \partial x, \\
& U_{y}=\partial \phi / \partial y .
\end{aligned}
$$

When the bosses are close together, a correction factor may be applied that is evaluated in Sec. VI as a measure of the nonradiative interaction.

Under the same assumptions, the divergence $D$ at $z=0$ may be written

$$
D=\nabla^{2} \phi .
$$

Hence,

$$
\begin{aligned}
\mu_{x} & =-\frac{1}{2} a^{3} N \partial \phi / \partial x, \\
\mu_{y} & =-\frac{1}{2} \alpha^{3} N \partial \phi / \partial y, \\
\mu & =\frac{1}{3} a^{3} N \nabla^{2} \phi .
\end{aligned}
$$

With these values, relation $4 \mathrm{~d}$ becomes

$$
\partial \phi / \partial z=\frac{3}{2} \tau\left(\partial^{2} \phi / \partial x^{2}+\partial^{2} \phi / \partial y^{2}\right)-\tau \nabla^{2} \phi,
$$

where

$$
\tau=(2 r / 3) a^{3} N,
$$

represents the volume of the bosses per unit area.

The problem of evaluating the acoustic reflection for a rough surface is now equivalent to solving the wave equation for $\phi$,

$$
\nabla^{2} \phi+k^{2} \phi=0,
$$

with the boundary condition $4 \mathrm{k}$ at $z=0$.
By taking into account Eq. 4m, Relation $4 \mathrm{k}$ may also be written in either form

$$
\partial \phi / \partial z=\frac{3}{2} \tau\left(\partial^{2} \phi / \partial x^{2}+\partial^{2} \phi / \partial y^{2}\right)+\tau k^{2} \phi,
$$

or

$$
\partial \phi / \partial z=-\frac{1}{2} \tau\left(k^{2} \phi+3 \partial^{2} \phi / \partial z^{2}\right) .
$$

Except for the numerical coefficients, the latter result is the same as derived previously. ${ }^{2}$ The difference is due to the addition of radiating sources.

\section{PLANE-WAVE REFLECTION WITH MULTIPLE SCATTER}

We apply the foregoing results to the reflection of a plane wave on a rough surface. The $z$ axis is normal to the reflecting surface. The incident plane wave propagates in a direction lying in the $x-z$ plane and the angle of incidence is denoted by $\alpha$. The incident wave is represented by the velocity potential

$$
\phi_{i}=A \exp (i z k \cos \alpha-i x k \sin \alpha),
$$

and the reflected wave by

$$
\phi_{r}=B \exp (-i z k \cos \alpha-i x k \sin \alpha),
$$

with complex amplitudes $A$ and $B$. The total velocity potential is

$$
\phi=\phi_{i}+\phi_{r} .
$$

We substitute this value into the boundary condition, Eq. 4o, at $z=0$. This yields the following relation between the coefficients $A$ and $B$ :

$$
B=A \frac{\cos \alpha-\frac{1}{2} i \tau k\left(1-3 \cos ^{2} \alpha\right)}{\cos \alpha+\frac{1}{2} i \tau k\left(1-3 \cos ^{2} \alpha\right)} .
$$

We may write this equation in the form

$$
B=A e^{2 i \psi},
$$

with

$$
\tan \psi=-\frac{1}{2} \tau k \sin ^{2} \alpha / \cos \alpha+\tau k \cos \alpha .
$$

The angle $2 \psi$ is the phase of the reflected wave relative to the incident wave.

The first term in Eq. $5 \mathrm{f}$ represents a phase retardation and as we shall see it is due essentially to the cooperative interaction of the roughness elements and the multiple scatter. The second term represents a phase gain

$$
2 \psi^{\prime}=2 \tau k \cos \alpha .
$$

Referring to Expressions $5 \mathrm{a}$ and $5 \mathrm{~b}$, we conclude that it is exactly the same as if specular reflection took place on a smooth plane that has been raised by an amount $z=\tau$ above the original level $z=0$. Since $\tau$ is the volume of roughness per unit area, this raised reflecting surface is obtained by spreading the volume of the roughness as a smooth layer of uniform thickness. 
At normal incidence, the first term in Eq. 5f disappears. Since $\psi$ is small, the actual phase gain is

$$
2 \psi=2 \psi^{\prime}=2 \tau k \text {. }
$$

Hence, in this case, the effect of the roughness is the same as raising the plane of specular reflection by an amount $\tau$.

There is an angle of incidence for which the two terms in Eq. $5 f$ cancel each other and the change of phase vanishes. The angle of incidence $\alpha$ at which this occurs, is obtained by putting $\psi=0$. Its value is given by

$$
\cos \alpha=1 / \sqrt{3}
$$

and is equal to about $55^{\circ}$.

For angles of incidence larger than this value the first term in Eq. 5f predominates and leads to a phase retardation that increases rapidly, and for $\alpha=\pi / 2$ tends to the limit

$$
2 \psi=-\pi .
$$

Hence, at grazing incidence the reflection occurs with a complete phase reversal causing the total field to vanish in the vicinity of the solid. This effect was already derived earlier ${ }^{1}$ in an incomplete theory, using only the induced dipoles. It was pointed out that it is due to a resonance between the incident wave and the accumulation of disturbances produced by the roughness and subject to multiple scatter interaction. It should be kept in mind that this grazing incidence phase reversal occurs no matter how small the roughness. The roughness size determines only how close we must approach grazing incidence in order to observe the reversal.

\section{SURFACE WAVES DUE TO ROUGHNESS}

Because of the presence of roughness, a wave may propagate along the surface. This can be shown by considering a velocity potential $\phi$ of the type

$$
\phi=A e^{-\beta z} e^{-i l x} \text {. }
$$

Again, the time factor $\exp (i \omega t)$ has been omitted. The solution must satisfy the wave equation (Eq. $4 \mathrm{~m}$ ); hence,

$$
\beta^{2}=l^{2}-k^{2} \text {. }
$$

For $l>k$, the value of $\beta$ may be chosen real positive and the field (Eq. 6a) dies out exponentially with the distance $z$ from the surface. The phase velocity along the surface is

$$
V=\omega / l=(k / l) c
$$

or

$$
V=c\left(1-\beta^{2} / l^{2}\right)^{\frac{1}{2}} .
$$

Hence, it is smaller than the velocity of sound.

The value of $\beta$ is obtained by substituting Expression 6a for $\phi$ into the boundary condition (Eq. 4o) at $z=0$.
Taking into account Relation $6 \mathrm{~b}$, we derive

$$
2 \tau \beta^{2}-2 \beta+\tau l^{2}=0,
$$

and

$$
\beta=\left[1 \pm\left(1-2 \tau^{2} l^{2}\right)^{\frac{1}{2}}\right] / 2 \tau .
$$

Validity of the theory requires the wavelength to be large relative to the roughness size. Hence, $\tau k \ll 1$ and the two values of $\beta$ are approximately

$$
\begin{aligned}
& \beta_{1} \cong 1 / \tau \\
& \beta_{2} \cong \tau l^{2} / 2 .
\end{aligned}
$$

The value $\beta_{1}$ must be excluded because it implies an imaginary value of the phase velocity (Eq. 6d) as can be seen by writing

$$
\beta_{1} / l=1 / \tau l \gg 1 .
$$

With the second value $\beta_{2}$ for $\beta$, the phase velocity becomes

$$
V=c\left(1-\frac{1}{4} \tau^{2} l^{2}\right)^{\frac{1}{2}} .
$$

Hence, $V$ depends on the wavelength $2 \pi / l$ and the propagation is slightly dispersive.

\section{NONRADIATIVE INTERACTION}

When the distance between hemispherical bosses is reduced, there comes a moment when their mutual nonradiative interaction must be taken into account. This interaction is caused mainly by the dipoles. This can be seen if we consider one hemispherical boss located at a certain point $P$. The velocities induced by the sources associated with the surrounding bosses tend to cancel out, while the velocities due to the dipoles tend to add up. This effect was evaluated in an earlier paper. ${ }^{1}$ If we denote by $U_{x}$ and $U_{y}$ the uncorrected velocity components as defined above in Sec. 3 and by $U_{x}{ }^{\prime}$ and $U_{y}{ }^{\prime}$, the velocity components, taking into account the nonradiative interactions of the bosses, we derive

$$
\begin{aligned}
& \kappa U_{x}^{\prime}=U_{x}, \\
& \kappa U_{y}^{\prime}=U_{y},
\end{aligned}
$$

where

$$
\kappa=1+\pi^{2} a^{3} / 4 b^{3} .
$$

In this expression, $b$ is the average distance between boss centers, and it is assumed here that the distribution is isotropic in all directions in the plane of reflection. In this case, the coefficient $\kappa$ measures a shadow effect that reduces the actual velocity $U_{x}{ }^{\prime}, U_{y}{ }^{\prime}$ as compared to the uncorrected values $U_{x}, U_{y}$ by a factor $1 / \kappa$.

The dipole-moment densities induced by the corrected velocities $U_{x}^{\prime} U_{y}^{\prime}$ are obtained by substituting these values of the velocity in Expressions $4 \mathrm{~g}$. We derive

$$
\begin{aligned}
& \mu_{x}=-\frac{1}{2} a^{3} N U_{x}{ }^{\prime}=-(1 / 2 \kappa) a^{3} N U_{x}, \\
& \mu_{y}=-\frac{1}{2} a^{3} N U_{y}{ }^{\prime}=-(1 / 2 \kappa) a^{3} N U_{y} .
\end{aligned}
$$


The source density $\mu$ is unaffected. Proceeding as in Sec. III we obtain the boundary condition

$$
\partial \phi / \partial z=\frac{3}{2}(\tau / \kappa)\left(\partial^{2} \phi / \partial x^{2}+\partial^{2} \phi / \partial y^{2}\right)+\tau k^{2} \phi .
$$

This condition includes the nonradiative interaction represented by the factor $\kappa$. Since this factor depends on $(a / b)^{3}$, the effect vanishes rapidly for distances between bosses that exceed a few radii.

By including this factor $\kappa$, the theory is applicable to cases where the distances between bosses is comparable to their radii. We shall refer to $\kappa$ as the close interaction factor. As already pointed out for an isotropic distribution of bosses this close interaction corresponds to a mutual shielding or shadow effect and a value of $1 / \kappa$ smaller than one. In general, the effect does not always amount to a shielding. For example, if the distribution of bosses is not isotropic they may be close together only in one direction. For a velocity perpendicular to this direction, the close interaction factor will give a value of $1 / \kappa$ larger than one. Hence, there will be two values of $\kappa$ attached to two mutually perpendicular directions, resulting in anisotropic properties of the reflection. This will be analyzed further in Sec. 9.

\section{SHAPE FACTOR}

Until now, the roughness has been represented by bosses of hemispherical shape. However, this restriction is not necessary and the method may readily be extended to arbitrary shapes. As an example, let us choose a needle-shaped protuberance perpendicular to the surface. We first consider the case where the protuberances are isotropic; i.e., they are bodies of revolution about an axis perpendicular to the surface. The nonisotropic case is discussed in Sec. IX.

We denote again by $U_{x}$ and $U_{y}$ the velocity at the location of the needle when the latter is removed. The presence of the needle induces radiating dipoles distributed along its axis as can be derived by applying the theory of diffraction by a cylindrical obstacle. At a certain distance from the needle, the integrated effect is approximately the same as that obtained from a single dipole at the surface of reflection. The magnitude of this dipole as related to the velocity $U_{x}, U_{y}$ is different from that of the hemispherical boss. This may be formulated by generalizing Expressions $4 \mathrm{~g}$ for the dipole densities writing them in the form

$$
\begin{aligned}
& \mu_{x}=-(3 \tau / 4 \pi) \sigma U_{x}, \\
& \mu_{y}=-(3 \tau / 4 \pi) \sigma U_{y},
\end{aligned}
$$

where $\tau$ now denotes the volume of the protuberances per unit area. The influence of the shape is represented by a shape factor $\sigma$. For the particular case of hemispherical bosses, $\sigma=1$, and $\tau$ is given by Eq. 4l. For sharp roughness, $\sigma>1$, and for flat roughness, $\sigma<1$.
We may also include the nonradiative interference. This is obtained by writing

$$
\begin{aligned}
& \mu_{x}=-(3 \tau / 4 \pi) \sigma U_{x}^{\prime}=-(3 / 4 \pi) \tau(\sigma / k) U_{x}, \\
& \mu_{y}=-(3 \tau / 4 \pi) \sigma U_{y}^{\prime}=-(3 / 4 \pi) \tau(\sigma / k) U_{y},
\end{aligned}
$$

using the values obtained from Eq. 7a for $U_{x}^{\prime}$ and $U_{y}^{\prime}$. The source density $\mu$ depends only on the volume $\tau$ and is written as before

$$
\mu=(1 / 2) \pi \tau D .
$$

These results combined with Eqs. 4d, 4h, 4i, and $4 \mathrm{~m}$ lead to the following boundary condition:

with

$$
\partial \phi / \partial z=\frac{3}{2} \gamma \tau\left(\partial^{2} \phi / \partial x^{2}+\partial^{2} \phi / \partial y^{2}\right)+\tau k^{2} \phi,
$$

$$
\gamma=\sigma / \kappa .
$$

In this equation, the parameters are $\tau$ the volume of protuberances per unit area; and $\gamma$, which represents the combined influence of the shape factor $\sigma$ and the close interaction factor $\kappa$. We refer to $\gamma$ as the geometric factor.

\section{NONUNIFORM ROUGHNESS}

Heretofore, it was assumed that $\tau$, the volume of the roughness per unit area, as well as the shape and close interaction factors $\sigma$ and $\kappa$ are constant. In order to extend the theory to nonuniform roughness, we must distinguish between two types of nonuniformity.

Consider first the local geometry restricted to an averaging domain that is small relative to the wavelength. Within this domain, we may assume that the protuberances vary in size. For example, hemispherical bosses of various radii $a_{i}$ may be distributed in a certain way over the unit area. In the absence of nonradiative interaction equations, (Eqs. $4 \mathrm{~g}$ ), expressing the dipole and source densities, may be generalized as follows:

$$
\begin{aligned}
& \mu_{x}=-\frac{1}{2} U_{x} \sum^{i} a_{i}{ }^{3}=-\frac{3 \tau}{4 \pi} U_{x}, \\
& \mu_{y}=-\frac{1}{2} U_{y} \sum^{i} a_{i}{ }^{3}=-\frac{3 \tau}{4 \pi} U_{y}, \\
& \mu=\frac{1}{3} D \sum^{i} a_{i}{ }^{3}=\frac{\tau}{2 \pi} D .
\end{aligned}
$$

The summation is extended to all the bosses over the unit area. The value of $\tau$ as before represents the volume of the bosses per unit area. It is defined as

where

$$
\tau=\sum^{i} \tau_{i}
$$

$$
\tau_{i}=\frac{2}{3} \pi a_{i}{ }^{3}
$$


represents the volume of each hemispherical boss of radius $a_{i}$.

If nonradiative interaction is present, each boss is associated with a close interaction factor $\kappa_{i}$. In addition, the bosses may be replaced by protuberances of various shapes each associated with a shape factor $\sigma_{i}$. We assume isotropic properties of the roughness. This amounts to saying that $\kappa_{i}$ and $\sigma_{i}$ are the same for all directions.

In this case, it is possible to generalize the definition of the geometric factor $\gamma$ (Eq. 8e) by writing

$$
\tau \gamma=\sum^{i} \tau_{i}\left(\sigma_{i} / \kappa_{i}\right)
$$

This factor $\gamma$ is a weighted local average of the shape and close interaction factors. The dipole densities may then be written

$$
\begin{aligned}
& \mu_{x}=-(3 / 4 \pi) \gamma \tau U_{x}, \\
& \mu_{y}=-(3 / 4 \pi) \gamma \tau U_{y} .
\end{aligned}
$$

As before, $\tau$ is the volume of the roughness per unit area. The value of $\mu$ is unaffected by shape and nonradiative interaction and remains as given by the third of Eqs. 9a.

The second type of nonuniformity that we now consider is quite different. It is represented by a continuous variation of the parameters $\tau$ and $\gamma$ over the reflecting surface. Hence, they are functions of $x$ and $y$ :

$$
\begin{gathered}
\tau=\tau(x, y), \\
\gamma=\gamma(x, y) .
\end{gathered}
$$

This means that the local average size and average geometry are not uniform. However, applicability of the theory requires that the distributions $\tau(x, y)$ and $\gamma(x, y)$ be sufficiently smooth. More precisely, they must contain only wavelengths that are sufficiently large relative to the size of the roughness. Using the values obtained from Eq. 9e for $\mu_{x}$ and $\mu_{y}$ and the third of Eqs. 9a for $\mu$, Relation 4d becomes

$$
\frac{\partial \phi}{\partial z}=\frac{3}{2} \frac{\partial}{\partial x}\left(\gamma \tau \frac{\partial \phi}{\partial x}\right)+\frac{3}{2} \frac{\partial}{\partial y}\left(\begin{array}{c}
\gamma \tau \frac{\partial \phi}{\partial y} \\
\gamma
\end{array}\right)+\tau k^{2} \phi .
$$

This is the boundary condition for nonuniform roughness.

Since $\gamma \tau$ may be represented by a spectral distribution, this result opens the way to an analysis of multiple scatter including a noncoherent reflection due to nonuniformity of the roughness.

\section{ANISOTROPIC ROUGHNESS}

Assume the bosses to be half-ellipsoids lying with the flat sides on the plane of reflection and their axes parallel to the $x$ and $y$ directions. The induced dipoles are different in these two directions. We write

$$
\begin{aligned}
& \mu_{x}=-(3 / 4 \pi) \gamma_{x x} \tau U_{x}, \\
& \mu_{y}=-(3 / 4 \pi) \gamma_{y y} \tau U_{y} .
\end{aligned}
$$

The geometric factors $\gamma_{x x}$ and $\gamma_{y y}$ are different in the $x$ and $y$ directions. They are defined by equations similar to Eq. 9d:

$$
\begin{array}{r}
\tau \gamma_{x x}=\sum \sum_{\kappa_{x i}^{i}}^{\tau_{i}}, \\
\tau \gamma_{y y}=\sum \sum_{\kappa_{x i}}^{\tau_{\kappa_{y i}}},
\end{array}
$$

where $\sigma_{x i}$ and $\kappa_{x i}$ are shapes and close interaction factors of the ellipsoidal bosses, in the $x$ direction, with corresponding designations $\sigma_{y_{i}}$ and $\kappa_{y i}$ of these factors in the $y$ direction. As before, $\tau$ denotes the volume of the bosses per unit area and $\tau_{i}$ the volume of individual bosses. The geometric factor is obviously a two-dimensional symmetric tensor $\gamma_{k j}=\gamma_{j k}$ and in general we may write Eq. 10b in the form

$$
\mu_{k}=-(3 / 4 \pi) \tau \gamma_{k l} U_{l},
$$

with the usual summation convention. The value of $\mu$ is the same as given by the third of Eqs. 9a. By introducing these results in Eq. $4 \mathrm{~d}$ we obtain

$$
\partial \phi / \partial z=\frac{3}{2}\left(\partial / \partial x_{k}\right)\left(\tau \gamma_{k l} \partial \phi / \partial x_{l}\right)+\tau k^{2} \phi .
$$

We designate by $x_{1}$ and $x_{2}$ the $x$ and $y$ coordinates.

Note that hemispherical bosses may give rise to anisotropic properties if, for example, in the $x$ direction they are closer together than in the $y$ direction. In this case, the shape factors are $\sigma_{x i}=\sigma_{y i}=1$. But if the bosses are close enough along $x$, the nonradiative interaction yields two different coefficients $\kappa_{x i}$ and $\kappa_{y i}$; hence, $\gamma_{x x}$ and $\gamma_{y y}$ will be different.

The boundary condition (Eq. 10d) is obviously quite general and applies to bosses that are not ellipsoidal. It generalizes Eq. $9 \mathrm{~g}$ to the case where in addition to the nonuniformity of size and shape the directions of the local axes of anisotropy vary continuously over the surface of reflection.

\section{ACKNOWLEDGMENT}

This work was prepared for the Bell Telephone Laboratories, Incorporated, under contract DA-30-069 -AMC-333(Y). 